\begin{tabular}{|c|l|}
\hline Title & Weighted Strichartz estimates for the wave equation in even space dimensions \\
\hline Author(s) & Kato, Jun; Ozawa, Tohru \\
\hline Citation & Hokkaido University Preprint Series in Mathematics, 603, 1-20 \\
\hline Issue Date & 2003 \\
\hline DOI & 10.14943/83748 \\
\hline Doc URL & http://hdl.handle.net/2115/69352 \\
\hline Type & bulletin (article) \\
\hline File Information & pre603.pdf \\
\hline
\end{tabular}

Instructions for use 


\title{
Weighted Strichartz estimates for the wave equation in even space dimensions
}

Dedicated to Professor Mitsuru Ikawa on the occasion of his sixtieth birthday

\author{
Jun Kato*† and Tohru Ozawa \\ Department of Mathematics, Hokkaido University \\ Sapporo 060-0810, Japan
}

\begin{abstract}
We prove the weighted Strichartz estimates for the wave equation in even space dimensions with radial symmetry in space. Although the odd space dimensional cases have been treated in our previous paper [4], the lack of the Huygens principle prevents us from a similar treatment in even space dimensions. The proof is based on the two explicit representations of solutions due to Rammaha [10] and Takamura [13] and to Kubo-Kubota [5]. As in the odd space dimensional cases [4], we are also able to construct self-similar solutions to semilinear wave equations on the basis of the weighted Strichartz estimates.
\end{abstract}

\section{Introduction and the main result}

This paper is a sequel to [4], where we study the weighted Strichartz estimates for the wave equation without the support condition and self-similar solutions to nonlinear wave equations. The results in [4] are restricted to odd space dimensions, for the proof of the weighted Strichartz estimates depends on an explicit representation

\footnotetext{
*JSPS Research Fellow

†kato@math.sci.hokudai.ac.jp
} 
formula of the free solutions which holds only for odd space dimensions. The corresponding explicit representation formula for even space dimensions causes a number of serious difficulties when one follows a similar method. One of the difficulties lies in the existence of region of diffusion of waves, where a similar technique seems useless to control the tail of waves which lives inside the light cone and has a singularity on the light cone. It seems technical as far as the difference of representation formula is concerned, while it looks essential because the situation depends heavily on the Huygens principle.

In this paper we prove the weighted Strichartz estimates for the wave equation in even space dimensions. Let $F$ be a function on $\mathbf{R}_{+}^{1+n}=(0, \infty) \times \mathbf{R}^{n}$ with radial symmetry in space and let $w$ be a solution of the wave equation

$$
\begin{aligned}
& \square w=F, \quad(t, x) \in \mathbf{R}_{+}^{1+n}, \\
& \left.w\right|_{t=0}=\left.\partial_{t} w\right|_{t=0}=0, \quad x \in \mathbf{R}^{n},
\end{aligned}
$$

where $\square=\partial_{t}^{2}-\Delta$ is the d'Alembertian with Laplacian $\Delta$ in $\mathbf{R}^{n}$.

Theorem 1. Let $n \geq 2$ be even and let $2<q<\frac{2(n+1)}{n-1}$. Let a and $b$ satisfy

$$
a-b+\frac{n+1}{q}=\frac{n-1}{2}, \quad \frac{n}{q}-\frac{n-1}{2}<b<\frac{1}{q} .
$$

Then, there exists a constant $C>0$ such that

$$
\left\|\left.\left.\left|t^{2}-\right| x\right|^{2}\right|^{a} w\right\|_{L^{q}\left(\mathbf{R}_{+}^{1+n}\right)} \leq C\left\|\left.\left.\left|t^{2}-\right| x\right|^{2}\right|^{b} F\right\|_{L^{q^{\prime}\left(\mathbf{R}_{+}^{1+n}\right)}} .
$$

Remark 1.1. (1) Theorem 1 also holds when $n$ is odd. See [3, Lemma 3.1].

(2) A similar estimate to Theorem 1 has been shown by Georgiev-Lindblad-Sogge [1, Theorem 1.4] in odd space dimensions, and they announced that the corresponding even space dimensional cases also hold. In the above theorem their support condition $\operatorname{supp} F \subset\{(t, x) ;|x|<t\}$ is removed at the cost of an additional lower bound $b>\frac{n}{q}-\frac{n-1}{2}$.

(3) As for the weighted Strichartz estimates without radial symmetry, see [1], [14], which require that the support of $F$ is contained in the light cone.

The proof of Theorem 1 is based on explicit representations of the solution $w$ derived by using the radial symmetry of the inhomogeneous term $F$. Such representation and duality arguments enables us to reduce the weighted Strichartz estimates to the weighted Hardy-Littlewood-Sobolev inequality, as in the odd space dimensional cases [4]. As compared with the odd dimensional cases, the lack of the 
Huygens principle makes the treatment of the even dimensional cases more difficult. To overcome such difficulties we divide the proof of Theorem 2 into two cases, $2<q \leq \frac{2(n-1)}{n-2}, \frac{2(n-1)}{n-2}<q<\frac{2(n+1)}{n-1}$, and in each case we apply different representation of the solution, which is due to Rammaha [10] and Takamura [13] and to Kubo-Kubota [5], respectively.

Theorem 1 has an application to the existence of self-similar solutions to the Cauchy problem for semilinear wave equations of the form

$$
\begin{aligned}
& \square u=f(u), \quad(t, x) \in \mathbf{R}^{n}, \\
& \left.u\right|_{t=0}=\varepsilon \phi,\left.\quad \partial_{t} u\right|_{t=0}=\varepsilon \psi, \quad x \in \mathbf{R}^{n},
\end{aligned}
$$

where $\varepsilon>0$ is a small parameter and $f(u)$ is homogeneous of degree $p$ with respect to $u$ and satisfies the estimates

$$
\begin{gathered}
|f(u)| \leq C|u|^{p}, \\
|f(u)-f(v)| \leq C\left(|u|^{p-1}+|v|^{p-1}\right)|u-v|,
\end{gathered}
$$

where $C$ is independent of $u$ and $v$, and $p>1$. A solution $u$ of (1.4) is called a self-similar solution if

$$
\lambda^{2 /(p-1)} u(\lambda t, \lambda x)=u(t, x)
$$

for all $\lambda>0$.

Regarding the existence of self-similar solutions to the Cauchy problem (1.4), (1.5), several results are known. First, Pecher [7] showed the existence of self-similar solutions for $p>(4+\sqrt{13}) / 3$ when $n=3$. This lower bound on $p$, which is denoted by $p_{1}(n)$ in general dimensions $n$, is the one that appeared in Mochizuki-Motai [6] in connection with the scattering theory. Specifically, $p_{1}(n)$ is given by the positive root of the following quadratic equation in $p$ :

$$
n(n-1) p^{2}-\left(n^{2}+3 n-2\right) p+2=0 .
$$

Pecher's result is extended for general dimensions by Ribaud-Youssfi [11].

Next, Pecher [8] also showed the existence of self-similar solutions for $1+\sqrt{2}<$ $p \leq 2$ when $n=3$ and gave a counter-example indicating that the lower bound on $p$ is sharp. This lower bound, which is denoted by $p_{0}(n)$ in general dimensions $n$, is known as the critical exponent concerning the existence of global solutions for compactly supported, smooth, small data. Specifically, $p_{0}(n)$ is given by the positive root of the following quadratic equation in $p$ :

$$
(n-1) p^{2}-(n+1) p-2=0 .
$$


Note that $p_{0}(n)<p_{1}(n)$ holds in all dimensions. Hidano [2] also showed the existence of self-similar solutions for $p_{0}(n)<p<\frac{n+3}{n-1}$ when $n=2,3$.

In our previous work [4], we proved the existence of radially symmetric selfsimilar solutions for $p_{0}(n)<p<\frac{n+3}{n-1}$ in odd space dimensions under the data of the form

$$
\phi(x)=C_{1}|x|^{-\frac{2}{p-1}}, \quad \psi(x)=C_{2}|x|^{-\frac{2}{p-1}-1},
$$

where $C_{1}, C_{2} \in \mathbf{R}$. The data (1.6) is the same as (3) in Pecher [7]. Moreover, the data (1.6) has the critical decay rate at infinity in space (see Takamura [13] for example).

To state our result on the existence of self-similar solutions to (1.4), we introduce weak Lebesgue spaces. Weak Lebesgue spaces $L_{w}^{p}$ are defined by

$$
L_{w}^{p}=\left\{f \in L_{\mathrm{loc}}^{1} ;\|f\|_{L_{w}^{p}} \equiv \sup _{\lambda>0} \lambda|\{x ;|f(x)|>\lambda\}|^{1 / p}<\infty\right\},
$$

for $1 \leq p<\infty$, where $|\cdot|$ denotes the Lebesgue measure. Although $\|\cdot\|_{L_{w}^{p}}$ does not satisfy the triangle inequality, there exists a norm equivalent to $\|\cdot\|_{L_{w}^{p}}$ for $p>1$ and with this norm the space $L_{w}^{p}$ becomes a Banach space.

Theorem 2. Let $n \geq 2$ be even and let $p_{0}(n)<p<\frac{n+3}{n-1}$. Then, there exists a unique solution $u$ of the integral equation associated with the Cauchy problem (1.4), (1.5) with (1.6) such that

$$
\left.\left.\left|t^{2}-\right| x\right|^{2}\right|^{\gamma} u \in L_{w}^{p+1}\left(\mathbf{R}_{+}^{1+n}\right)
$$

if $\varepsilon>0$ is sufficiently small, where $\gamma=\frac{1}{p-1}-\frac{n+1}{2(p+1)}$.

The norm of the weighted weak Lebesgue space to which the solution $u$ belongs is invariant with respect to the scale transform $u \longmapsto u_{\lambda}$. This invariance is important to treat self-similar solutions and requires a direct use of the weight of homogeneous type. Since self-similar solutions $u$ of (1.4) are to be homogeneous functions in time and space variables by the definition, we observe that $\left.\left.\left|t^{2}-\right| x\right|^{2}\right|^{\gamma} u$ does not belong to the usual Lebesgue spaces on $\mathbf{R}_{+}^{1+n}$, and therefore it is natural to use weak Lebesgue spaces instead.

The corresponding result in odd space dimensions have been obtained in [4] and the restriction to such space dimensions only comes from the weighted Strichartz estimates, which was the key estimate to construct the solution. Thus, once we obtain the weighted Strichartz estimates in even space dimensions, namely, Theorem 
1 in this paper, Theorem 2 is proved by the same arguments as in [4] and we omit it here.

This paper is organized as follows. In Section 2 we prove Theorem 1 in the case where $n \geq 4,2<q \leq \frac{2(n-1)}{n-2}$. In Section 3 we prove Theorem 1 in the case where $n \geq 4, \frac{2(n-1)}{n-2}<q<\frac{2(n+1)}{n-1}$. In Section 4 we prove Theorem 1 in the case $n=2$.

\section{Proof of Theorem 1 in the case $2<q \leq \frac{2(n-1)}{n-2}$}

In this section we prove Theorem 1 in the case where $n \geq 4$ is even and $2<q \leq$ $\frac{2(n-1)}{n-2}$. The proof is based on the following representation and estimate of free solutions. In the sequel we denote $F$ with radial symmetry in space as a function on $(0, \infty) \times(0, \infty)$.

Lemma 2.1 (Rammaha [10], Takamura [13]). Let $n \geq 2$ be even. Then,

$$
\begin{aligned}
w(t, r)= & r^{-\frac{n}{2}+1} \int_{0}^{t} \int_{|t-s-r|}^{t-s+r} \lambda^{\frac{n}{2}} L(\lambda, r, t-s) F(s, \lambda) d \lambda d s \\
& +r^{-\frac{n}{2}+1} \int_{0}^{\max (t-r, 0)} \int_{0}^{t-s-r} \lambda^{\frac{n}{2}} \widetilde{L}(\lambda, r, t-s) F(s, \lambda) d \lambda d s,
\end{aligned}
$$

where $r=|x|, L$ and $\widetilde{L}$ satisfy the following estimates

$$
\begin{aligned}
& |L(\lambda, r, \tau)| \leq\left\{\begin{array}{r}
C r^{-\frac{1}{2}} \lambda^{-\frac{1}{2}}, \quad \text { if } 0<\tau \leq r, r-\tau<\lambda<r+\tau, \\
C_{\sigma} \lambda^{-\frac{1}{2}+\sigma}(r+\lambda-\tau)^{-\sigma}(r+\lambda+\tau)^{-\sigma}(\tau+r-\lambda)^{-\frac{1}{2}+\sigma}, \\
\text { if } 0<r<\tau, \tau-r<\lambda<r+\tau,
\end{array}\right. \\
& |\widetilde{L}(\lambda, r, \tau)| \leq C_{\sigma} r^{-\frac{1}{2}+\sigma} \lambda^{-\frac{1}{2}+\sigma}(\tau-r-\lambda)^{-\sigma}(\tau+r+\lambda)^{-\sigma}, \quad 0<\lambda<\tau-r,
\end{aligned}
$$

Remark 2.1. As we shall see below, $L$ and $\widetilde{L}$ are given explicitly by

$$
L(\lambda, r, \tau)=\frac{2}{\pi} \int_{|r-\lambda|}^{\tau} \frac{\rho T_{\frac{n-2}{2}}\left(\left(\lambda^{2}+r^{2}-\rho^{2}\right) / 2 r \lambda\right)}{\sqrt{\tau^{2}-\rho^{2}} \sqrt{\rho^{2}-(r-\lambda)^{2}} \sqrt{(r+\lambda)^{2}-\rho^{2}}} d \rho
$$

for $|\tau-r|<\lambda<\tau+r$, and

$$
\widetilde{L}(\lambda, r, \tau)=\frac{2}{\pi} \int_{|r-\lambda|}^{r+\lambda} \frac{\rho T_{\frac{n-2}{2}}\left(\left(\lambda^{2}+r^{2}-\rho^{2}\right) / 2 r \lambda\right)}{\sqrt{\tau^{2}-\rho^{2}} \sqrt{\rho^{2}-(r-\lambda)^{2}} \sqrt{(r+\lambda)^{2}-\rho^{2}}} d \rho,
$$

for $0<\lambda<\tau-r$, where $T_{k}$ denotes the Tschebyscheff polynomial of degree $k$ defined by

$$
T_{k}(z)=\frac{(-1)^{k}}{(2 k-1) ! !}\left(1-z^{2}\right)^{\frac{1}{2}} \frac{d^{k}}{d z^{k}}\left(1-z^{2}\right)^{k-\frac{1}{2}}
$$


Proof. This lemma is based on the representation due to Rammaha [10], Takamura [13]. We refer to [13, Lemma 2.3] on the representation of the following form,

$$
\begin{aligned}
w(t, r)= & \frac{2}{\pi} r^{-\frac{n}{2}+1} \int_{0}^{t} \int_{0}^{t-s} \frac{\rho}{\sqrt{(t-s)^{2}-\rho^{2}}} \\
& \times\left(\int_{|r-\rho|}^{r+\rho} \frac{T_{\frac{n-2}{2}}\left(\left(\lambda^{2}+r^{2}-\rho^{2}\right) / 2 r \lambda\right)}{\sqrt{\lambda^{2}-(r-\rho)^{2}} \sqrt{(r+\rho)^{2}-\lambda^{2}}} \lambda^{\frac{n}{2}} F(s, \lambda) d \lambda\right) d \rho d s,
\end{aligned}
$$

where we have used the Duhamel principle to translate the representation on the solution of the homogeneous equation to the one of the inhomogeneous equation. Changing the order of integrals with respect to $\rho$ and $\lambda$ in (2.4) and changing the combination of pairs on the denominator, we obtain the representation (2.1) with $L$ and $\widetilde{L}$ given by $(2.2)$ and $(2.3)$.

In the following, we give the estimate on $L$ and $\widetilde{L}$. To begin with, we notice that

$$
\left|T_{\frac{n-2}{2}}\left(\left(\lambda^{2}+r^{2}-\rho^{2}\right) / 2 r \lambda\right)\right| \leq 1
$$

if $\rho \geq|r-\lambda|$, since it is known that

$$
\left|T_{k}(z)\right| \leq 1 \quad \text { if }|z| \leq 1
$$

and $\rho \geq|r-\lambda|$ implies $\left|\left(\lambda^{2}+r^{2}-\rho^{2}\right) / 2 r \lambda\right| \leq 1$.

We first give the estimate on $L$ in the case $0 \leq r-\tau<\lambda<r+\tau$. By (2.5),

$$
\begin{aligned}
|L(\lambda, r, \tau)| & \leq C \int_{|r-\lambda|}^{\tau} \frac{\rho}{\sqrt{\tau^{2}-\rho^{2}} \sqrt{\rho^{2}-(r-\lambda)^{2}} \sqrt{(r+\lambda)^{2}-\rho^{2}}} d \rho \\
& \leq \frac{C}{\sqrt{(r+\lambda)^{2}-\tau^{2}}} \int_{|r-\lambda|}^{\tau} \frac{\rho}{\sqrt{\tau^{2}-\rho^{2}} \sqrt{\rho^{2}-(r-\lambda)^{2}}} d \rho \\
& =C(r+\lambda+\tau)^{-\frac{1}{2}}(r+\lambda-\tau)^{-\frac{1}{2}} \\
& \leq C r^{-\frac{1}{2}} \lambda^{-\frac{1}{2}}
\end{aligned}
$$

since $r-\tau \geq 0$. In the above estimate, we have used

$$
\int_{a}^{b} \frac{x}{\left(x^{2}-a^{2}\right)^{\alpha}\left(b^{2}-x^{2}\right)^{\beta}} d x=\frac{1}{2} B(1-\alpha, 1-\beta)\left(b^{2}-a^{2}\right)^{1-\alpha-\beta},
$$

for $\alpha, \beta<1, a<b$, where $B$ is the beta function.

We next give the estimate on $L$ in the case $0<\tau-r<\lambda<r+\tau$. In this case,

$$
|L(\lambda, r, \tau)| \leq \frac{C}{\left((r+\lambda)^{2}-\tau^{2}\right)^{\sigma}} \int_{|r-\lambda|}^{\tau} \frac{\rho}{\left(\tau^{2}-\rho^{2}\right)^{1-\sigma} \sqrt{\rho^{2}-(r-\lambda)^{2}}} d \rho
$$


for $0<\sigma \leq 1 / 2$, since $r+\lambda>\tau$. Then applying (2.6), we obtain

$$
|L(\lambda, r, \tau)| \leq C_{\sigma}\left((r+\lambda)^{2}-\tau^{2}\right)^{-\sigma}\left(\tau^{2}-(r-\lambda)^{2}\right)^{-\frac{1}{2}+\sigma},
$$

from which we obtain the desired estimate using $\tau-r>0$.

We finally give the estimate on $\widetilde{L}$. Similarly as above,

$$
|\widetilde{L}(\lambda, r, \tau)| \leq \frac{C}{\left(\tau^{2}-(r+\lambda)^{2}\right)^{\sigma}} \int_{|r-\lambda|}^{r+\lambda} \frac{\rho}{\sqrt{\rho^{2}-(r-\lambda)^{2}}\left((r+\lambda)^{2}-\rho^{2}\right)^{1-\sigma}} d \rho,
$$

for $0<\sigma \leq 1 / 2$, since $\tau>r+\lambda$. Then applying (2.6), we obtain

$$
|\widetilde{L}(\lambda, r, \tau)| \leq C_{\sigma}\left(\tau^{2}-(r+\lambda)^{2}\right)^{-\sigma}\left((r+\lambda)^{2}-(r-\lambda)^{2}\right)^{-\frac{1}{2}+\sigma}
$$

from which we obtain the desired estimate.

Before describing the proof of Theorem 2, we prepare the weighted HardyLittlewood-Sobolev inequality of the following form.

Lemma 2.2 ([12]). Let $0<\lambda<n, 1<r, s<\infty$. Let $\alpha<n / s^{\prime}$ and $\beta<n / r^{\prime}$ with $\alpha+\beta \geq 0$ satisfy $1 / s+1 / r+(\lambda+\alpha+\beta) / n=2$. Then,

$$
\left|\int_{\mathbf{R}^{n}} \int_{\mathbf{R}^{n}} \frac{f(x) g(y)}{|x|^{\alpha}|x-y|^{\lambda}|y|^{\beta}} d x d y\right| \leq C\|f\|_{L^{s}\left(\mathbf{R}^{n}\right)}\|g\|_{L^{r}\left(\mathbf{R}^{n}\right)} .
$$

Proof of Theorem 2 in the case where $n \geq 4,2<q \leq \frac{2(n-1)}{n-2}$. By duality and radial symmetry, the estimate (1.3) is equivalent to

$$
\begin{aligned}
&\left|\int_{0}^{\infty} \int_{0}^{\infty}\right| t^{2}-\left.r^{2}\right|^{a} w(t, r) \Phi(t, r) r^{n-1} d r d t \mid \\
& \leq C\left\|\left|t^{2}-r^{2}\right|^{b} r^{\frac{n-1}{q^{\prime}}} F\right\|_{L^{q^{\prime}}}\left\|r^{\frac{n-1}{q^{\prime}}} \Phi\right\|_{L^{q^{\prime}}}
\end{aligned}
$$

for all $\Phi \in C_{0}^{\infty}((0, \infty) \times(0, \infty))$.

By (2.1) we observe that

$$
\begin{aligned}
& \int_{0}^{\infty} \int_{0}^{\infty}\left|t^{2}-r^{2}\right|^{a} w(t, r) \Phi(t, r) r^{n-1} d r d t \\
& =\left(\int_{0}^{\infty} \int_{0}^{\infty} \int_{\max (t-r, 0)}^{t} \int_{r-(t-s)}^{r+t-s}+\int_{0}^{\infty} \int_{0}^{t} \int_{0}^{t-r} \int_{t-s-r}^{t-s+r}\right) \\
& \quad \times r^{\frac{n}{2}} \lambda^{\frac{n}{2}}\left|t^{2}-r^{2}\right|^{a} L(\lambda, r, t-s) F(s, \lambda) \Phi(t, r) d \lambda d s d r d t \\
& +\int_{0}^{\infty} \int_{0}^{t} \int_{0}^{t-r} \int_{0}^{t-s-r} r^{\frac{n}{2}} \lambda^{\frac{n}{2}}\left|t^{2}-r^{2}\right|^{a} \widetilde{L}(\lambda, r, t-s) F(s, \lambda) \Phi(t, r) d \lambda d s d r d t
\end{aligned}
$$


and applying the estimates on $L$ and $\widetilde{L}$ in Lemma 2.1 with $\sigma=1 / 2$, the left hand side of (2.7) is bounded by

$$
\begin{aligned}
& \int_{0}^{\infty} \int_{0}^{\infty} \int_{\max (t-r, 0)}^{t} \int_{r-(t-s)}^{r+t-s} r^{\frac{n-1}{2}} \lambda^{\frac{n-1}{2}}\left|t^{2}-r^{2}\right|^{a}|F(s, \lambda)||\Phi(t, r)| d \lambda d s d r d t \\
& \quad+\int_{0}^{\infty} \int_{0}^{t} \int_{0}^{t-r} \int_{0}^{t-s+r} \frac{r^{\frac{n}{2}} \lambda^{\frac{n}{2}}\left|t^{2}-r^{2}\right|^{a}|F(s, \lambda)||\Phi(t, r)|}{\sqrt{r+\lambda-t+s} \sqrt{r+\lambda+t-s}} d \lambda d s d r d t \\
& \equiv I_{1}+I_{2}
\end{aligned}
$$

Estimate of $I_{1}$. Applying the following change of variables

$$
t+r=u, t-r=v, s+\lambda=\xi, s-\lambda=\eta,
$$

we observe that

$$
\begin{aligned}
I_{1}=C\left(\int_{0}^{\infty} \int_{0}^{u} \int_{v}^{u}\right. & \left.\int_{-\xi+2 v}^{v}+\int_{0}^{\infty} \int_{-u}^{0} \int_{-v}^{u} \int_{-\xi}^{v}\right) \\
& \times \frac{G(\xi, \eta) H(u, v)}{|u|^{-a}|v|^{-a}|u-v|^{\gamma}|\xi|^{b}|\eta|^{b}|\xi-\eta|^{\gamma}} d \eta d \xi d v d u
\end{aligned}
$$

where $\gamma=(n-1)(1 / 2-1 / q)$ and we have set

$$
\left|s^{2}-\lambda^{2}\right|^{b} \lambda^{\frac{n-1}{q^{\prime}}}|F(s, \lambda)|=G(\xi, \eta), \quad r^{\frac{n-1}{q^{\prime}}}|\Phi(t, r)|=H(u, v) .
$$

To estimate the integral kernel we use the following claim.

Claim 1. If $\eta<v<\xi<u$, then $|\xi-v||u-\eta| \leq 2|u-v||\xi-\eta|$.

Proof. When $u-v \leq \xi-\eta$, we have

$$
|\xi-v||u-\eta| \leq(\xi-v)(u-v+\xi-\eta) \leq 2(u-v)(\xi-\eta),
$$

since $-v+\xi>0$ and $u>\xi$. Similarly, when $\xi-\eta<u-v$, we have

$$
|\xi-v||u-\eta| \leq(\xi-v)(u-v+\xi-\eta) \leq 2(\xi-\eta)(u-v),
$$

since $-v+\xi>0$ and $v>\eta$.

In each integral domain of the right hand side of (2.10) the condition $\eta<v<$ $\xi<u$ holds, and thus by Claim 1

$$
I_{1} \leq C \int_{\mathbf{R}} \int_{\mathbf{R}} \frac{1}{|v|^{-a}|v-\xi|^{\gamma}|\xi|^{b}}\left(\int_{\mathbf{R}} \int_{\mathbf{R}} \frac{G(\xi, \eta) H(u, v)}{|u|^{-a}|u-\eta|^{\gamma}|\eta|^{b}} d \eta d u\right) d \xi d v .
$$


Then applying Lemma 2.2 repeatedly, we obtain

$$
I_{1} \leq C\|G\|_{L^{q^{\prime}}}\|H\|_{L^{q^{\prime}}}=C\left\|\left|t^{2}-r^{2}\right|^{b} r^{\frac{n-1}{q^{\prime}}} F\right\|_{L^{q^{\prime}}}\left\|r^{\frac{n-1}{q^{\prime}}} \Phi\right\|_{L^{q^{\prime}}} .
$$

In fact, substituting $\alpha=-a, \beta=b, \lambda=\gamma=(n-1)(1 / 2-1 / q), r=s=q^{\prime}$ in Lemma 2.2, we observe that the condition $1 / s+1 / r+\lambda+\alpha+\beta=2, \alpha<1 / s^{\prime}$, $\beta<1 / r^{\prime}$ implies $a-b+\frac{n+1}{q}=\frac{n-1}{2}, b>\frac{n}{q}-\frac{n-1}{2}, b<\frac{1}{q}$, respectively.

Estimate of $I_{2}$. Similarly, by change of variables (2.9) and substitution (2.11),

$$
\begin{aligned}
I_{2}=\int_{0}^{\infty} \int_{0}^{u} & \left(\int_{0}^{v} \int_{-\xi}^{\xi}+\int_{v}^{u} \int_{-\xi}^{2 v-\xi}\right) \\
& \times \frac{|u-v|^{\frac{1}{2}-\gamma}|\xi-\eta|^{\frac{1}{2}-\gamma} G(\xi, \eta) H(u, v)}{|u|^{-a}|v|^{-a}|\xi|^{b}|\eta|^{b} \sqrt{\xi-v} \sqrt{u-\eta}} d \eta d \xi d v d u
\end{aligned}
$$

where $\gamma=(n-1)(1 / 2-1 / q)$ as before. In the domain of integration of the first and the second terms on the right hand side of (2.12), the conditions

$$
-\xi<\eta<\xi<v<u, \quad-\xi<\eta<2 v-\xi<v<\xi<u,
$$

hold respectively. In both cases we observe that

$$
(u-v)^{\frac{1}{2}-\gamma} \leq(u-\eta)^{\frac{1}{2}-\gamma}, \quad(\xi-\eta)^{\frac{1}{2}-\gamma} \leq(2 \xi)^{\frac{1}{2}-\gamma},
$$

since $v>\eta,-\eta<\xi, \xi>0$, and $2<q<\frac{2(n-1)}{n-2}$ implies $0<\gamma \leq 1 / 2$. Thus,

$$
I_{2} \leq C \int_{\mathbf{R}} \int_{\mathbf{R}} \frac{1}{|v|^{-a}|v-\xi|^{\frac{1}{2}}|\xi|^{b-\frac{1}{2}+\gamma}}\left(\int_{\mathbf{R}} \int_{\mathbf{R}} \frac{G(\xi, \eta) H(u, v)}{|u|^{-a}|u-\eta|^{\gamma}|\eta|^{b}} d \eta d u\right) d \xi d v .
$$

Then, applying Lemma 2.2 as above, we have

$$
I_{2} \leq C \int_{\mathbf{R}} \int_{\mathbf{R}} \frac{\|G(\xi, \cdot)\|_{L^{q^{\prime}}}\|H(\cdot, v)\|_{L^{q^{\prime}}}}{|v|^{-a}|v-\xi|^{\frac{1}{2}}|\xi|^{b-\frac{1}{2}+\gamma}} d \xi
$$

We can also apply Lemma 2.2 for the above integral, because $0<\gamma \leq 1 / 2$, which is due to $2<q \leq \frac{2(n-1)}{n-2}$, assures $b-1 / 2+\gamma \leq b<1 / q$, and $-a+(b-1 / 2+\gamma)=$ $2 / q-1 / 2 \geq 0$. The other assumptions of Lemma 2.2 are easily follows. Thus we obtain

$$
I_{2} \leq C\|G\|_{L^{q^{\prime}}}\|H\|_{L^{q^{\prime}}}=C\left\|\left|t^{2}-r^{2}\right|^{b} r^{\frac{n-1}{q^{\prime}}} F\right\|_{L^{q^{\prime}}}\left\|r^{\frac{n-1}{q^{\prime}}} \Phi\right\|_{L^{q^{\prime}}} .
$$

Therefore, we obtain (2.7) and this completes the proof of Theorem 2 in the case where $n \geq 4,2<q \leq \frac{2(n-1)}{n-2}$. 


\section{Proof of Theorem 1 in the case $\frac{2(n-1)}{n-2}<q<\frac{2(n+1)}{n-1}$}

In this section we prove Theorem 1 in the case where $n \geq 4$ is even and $\frac{2(n-1)}{n-2}<$ $q<\frac{2(n+1)}{n-1}$. The proof is based on the following representation and estimate of free solutions.

Lemma 3.1 (Kubo-Kubota [5]). Let $n \geq 2$ be even. Then,

$$
\begin{aligned}
w(t, r)= & r^{-n+2} \int_{0}^{t} \int_{|t-s-r|}^{t-s+r} \lambda^{n-1} K_{\frac{n-2}{2}}(\lambda, r, t-s) F(s, \lambda) d \lambda d s \\
& +r^{-n+2} \int_{0}^{\max (t-r, 0)} \int_{0}^{t-s-r} \lambda^{n-1} \widetilde{K}_{\frac{n-2}{2}}(\lambda, r, t-s) F(s, \lambda) d \lambda d s,
\end{aligned}
$$

where $r=|x|$, and $K_{\frac{n-2}{2}}, \widetilde{K}_{\frac{n-2}{2}}$ satisfy the following estimates,

$$
\begin{aligned}
& \qquad\left|K_{\frac{n-2}{2}}(\lambda, r, \tau)\right| \leq C r^{\frac{n-3}{2}} \lambda^{-\frac{n-1}{2}} \min \left(r^{\frac{1}{2}}, \lambda^{\frac{1}{2}}\right)(\lambda-\tau+r)^{-\frac{1}{2}}, \quad|\tau-r|<\lambda<\tau+r, \\
& \qquad\left|\widetilde{K}_{\frac{n-2}{2}}(\lambda, r, \tau)\right| \leq C r^{\frac{n-3}{2}+\sigma}(\tau-r)^{-\frac{n-2}{2}-\sigma}(\tau-r-\lambda)^{-\frac{1}{2}}, \quad 0<\lambda<\tau-r, \\
& \text { for } \tau, r>0,0 \leq \sigma \leq 1 / 2 .
\end{aligned}
$$

Remark 3.1. (1) We notice that $K_{\frac{n-2}{2}}$ and $\widetilde{K}_{\frac{n-2}{2}}$ are given explicitly by

$$
K_{j}(\lambda, r, \tau)=\int_{\lambda}^{\tau+r} \frac{H_{j}(\rho, r, \tau)}{\sqrt{\rho^{2}-\lambda^{2}}} d \rho, \quad \widetilde{K}_{j}(\lambda, r, \tau)=\int_{\tau-r}^{\tau+r} \frac{H_{j}(\rho, r, \tau)}{\sqrt{\rho^{2}-\lambda^{2}}} d \rho,
$$

for $j \geq 0$. Here,

$$
H_{j}(\rho, r, \tau)=\left(D_{\rho^{2}}^{*}\right)^{j} H(r, \rho-\tau), \quad|\rho-\tau|<r,
$$

where $D_{\rho^{2}}^{*}=\frac{d}{d \rho}\left(-\frac{1}{2 \rho}\right) \cdot H(r, \rho)=\left(r^{2}-\rho^{2}\right)^{\frac{n-3}{2}}$.

(2) One of the different point between Lemma 2.1 and Lemma 3.1 is the power of $\lambda$ derived from the estimates of the second terms on the right hand side of (2.1) and (3.1), which enables us to control the integral kernel as we shall see below.

Proof. The representation (3.1) with (3.2) is due to Kubo-Kubota [5, Lemma 3.4]. The estimates on $K_{\frac{n-2}{2}}$ and $\widetilde{K}_{\frac{n-2}{2}}$ is also due to [5, Lemma 4.2], which states

$$
\begin{aligned}
& \left|K_{\frac{n-2}{2}}(\lambda, r, \tau)\right| \leq C r^{\frac{n-3}{2}+\mu} \lambda^{-\frac{n-2}{2}-\mu}(\lambda-\tau+r)^{-\frac{1}{2}}, \quad|\tau-r|<\lambda<\tau+r, \\
& \left|\widetilde{K}_{\frac{n-2}{2}}(\lambda, r, \tau)\right| \leq C r^{\frac{n-3}{2}+\mu}(\tau-r)^{-\frac{n-2}{2}-\mu}(\tau-r-\lambda)^{-\frac{1}{2}}, \quad 0<\lambda<\tau-r,
\end{aligned}
$$

holds for $\tau, r>0$, and $\mu=0$ or $1 / 2$. Thus, taking minimum or interpolating with respect $\mu$, we obtain the desired estimate. 
Proof of Theorem 2 in the case where $n \geq 4, \frac{2(n-1)}{n-2}<q<\frac{2(n+1)}{n-1}$. As in the preceding case, it is sufficient to prove the estimate (2.7). By (3.1) the left hand side of (2.7) is bounded by

$$
\begin{aligned}
& \int_{0}^{\infty} \int_{0}^{\infty} \int_{0}^{t} \int_{|t-s-r|}^{t-s+r} r \lambda^{n-1}\left|t^{2}-r^{2}\right|^{a}\left|K_{\frac{n-2}{2}}(\lambda, r, t-s)\right||F(s, \lambda)||\Phi(t, r)| d \lambda d s d r d t \\
& +\left(\int_{0}^{\infty} \int_{t / 3}^{t} \int_{0}^{t-r} \int_{0}^{t-s-r}+\int_{0}^{\infty} \int_{0}^{t / 3} \int_{0}^{t-r} \int_{0}^{t-s-r}\right) \\
& \quad \times r \lambda^{n-1}\left|t^{2}-r^{2}\right|^{a}\left|\widetilde{K}_{\frac{n-2}{2}}(\lambda, r, t-s)\right||F(s, \lambda)||\Phi(t, r)| d \lambda d s d r d t \\
& \equiv I_{1}+I_{2}+I_{3} .
\end{aligned}
$$

Estimate of $I_{1}$. Applying the estimate on $K_{\frac{n-2}{2}}$ in Lemma 3.1, we obtain

$$
\begin{aligned}
I_{1} \leq C \int_{0}^{\infty} \int_{0}^{\infty} \int_{0}^{t} \int_{|t-s-r|}^{t-s+r} r^{\frac{n-1}{2}} \lambda^{\frac{n-1}{2}}\left|t^{2}-r^{2}\right|^{a} \\
\times \frac{\min \left(r^{\frac{1}{2}}, \lambda^{\frac{1}{2}}\right)}{|\lambda-t+s+r|^{\frac{1}{2}}}|F(s, \lambda)||\Phi(t, r)| d \lambda d s d r d t \\
=C \int_{0}^{\infty} \int_{-u}^{u} \int_{|v|}^{u} \int_{-\xi}^{v} \frac{\min \left\{|u-v|^{\frac{1}{2}},|\xi-\eta|^{\frac{1}{2}}\right\} G(\xi, \eta) H(u, v)}{|u|^{-a}|v|^{-a}|u-v|^{\gamma}|\xi|^{b}|\eta|^{b}|\xi-\eta|^{\gamma}|\xi-v|^{\frac{1}{2}}} d \eta d \xi d v d u
\end{aligned}
$$

where we have used the change of variables (2.9) and the substitution (2.11), and $\gamma=(n-1)(1 / 2-1 / q)$ as before. To estimate the integral kernel we use the following claim.

Claim 2. If $\eta<v<\xi<u$, then for $\gamma>1 / 2$

$$
\frac{\min \left\{|\xi-\eta|^{\frac{1}{2}},|u-v|^{\frac{1}{2}}\right\}}{|u-v|^{\gamma}|\xi-\eta|^{\gamma}|\xi-v|^{\frac{1}{2}}} \leq \frac{2^{\gamma}}{|\xi-v|^{\gamma}|u-\eta|^{\gamma}} .
$$

Proof. When $u-v \leq \xi-\eta$, we observe that

$$
\begin{aligned}
(\xi-v)^{\gamma-\frac{1}{2}}(u-\eta)^{\gamma} & \leq(u-v)^{\gamma-\frac{1}{2}}(u-v+\xi-\eta)^{\gamma} \\
& \leq 2^{\gamma}(u-v)^{\gamma-\frac{1}{2}}(\xi-\eta)^{\gamma}
\end{aligned}
$$

since $\xi<u,-v+\xi>0$. This implies

$$
\frac{|u-v|^{\frac{1}{2}}}{|u-v|^{\gamma}|\xi-\eta|^{\gamma}|\xi-v|^{\frac{1}{2}}} \leq \frac{2^{\gamma}}{|\xi-v|^{\gamma}|u-\eta|^{\gamma}} .
$$

Similarly, when $\xi-\eta<u-v$,

$$
\begin{aligned}
(\xi-v)^{\gamma-\frac{1}{2}}(u-\eta)^{\gamma} & \leq(\xi-\eta)^{\gamma-\frac{1}{2}}(u-v+\xi-\eta)^{\gamma} \\
& \leq 2^{\gamma}(\xi-\eta)^{\gamma-\frac{1}{2}}(u-v)^{\gamma}
\end{aligned}
$$


since $-v<-\eta,-v+\xi>0$. This implies

$$
\frac{|\xi-\eta|^{\frac{1}{2}}}{|u-v|^{\gamma}|\xi-\eta|^{\gamma}|\xi-v|^{\frac{1}{2}}} \leq \frac{2^{\gamma}}{|\xi-v|^{\gamma}|u-\eta|^{\gamma}} .
$$

This completes the proof of Claim 2.

Since the condition $\eta<v \leq|v|<\xi<u$ holds in the domain of integration of the above integral, and $\frac{2(n-1)}{n-2}<q<\frac{2(n+1)}{n-1}$ implies $\frac{1}{2}<\gamma<\frac{n-1}{n+1}$, we apply Claim 2 to have

$$
I_{1} \leq C \int_{\mathbf{R}} \int_{\mathbf{R}} \frac{1}{|v|^{-a}|v-\xi|^{\gamma}|\xi|^{b}}\left(\int_{\mathbf{R}} \int_{\mathbf{R}} \frac{G(\xi, \eta) H(u, v)}{|u|^{-a}|u-\eta|^{\gamma}|\eta|^{b}} d \eta d u\right) d \xi d v .
$$

Thus, applying Lemma 2.2 we obtain

$$
I_{1} \leq C\|G\|_{L^{q^{\prime}}}\|H\|_{L^{q^{\prime}}}=C\left\|\left|t^{2}-r^{2}\right|^{b} r^{\frac{n-1}{q^{\prime}}} F\right\|_{L^{q^{\prime}}}\left\|r^{\frac{n-1}{q^{\prime}}} \Phi\right\|_{L^{q^{\prime}}} .
$$

Estimate of $I_{2}$. Applying the estimate on $\widetilde{K}_{\frac{n-2}{2}}$ in Lemma 3.1 with $\sigma=0$, we obtain

$$
\begin{aligned}
I_{2} \leq C \int_{0}^{\infty} & \int_{t / 3}^{t} \int_{0}^{t-r} \int_{0}^{t-s-r} r^{-\frac{n-1}{2}} \lambda^{n-1}\left|t^{2}-r^{2}\right|^{a} \\
& \times(t-s-r)^{-\frac{n-2}{2}}(t-s-r-\lambda)^{-\frac{1}{2}}|F(s, \lambda)||\Phi(t, r)| d \lambda d s d r d t \\
=C \int_{0}^{\infty} & \int_{0}^{u / 2} \int_{0}^{v} \int_{-\xi}^{\xi} \\
& \times \frac{|\xi-\eta|^{\frac{n-1}{q}} G(\xi, \eta) H(u, v)}{|u|^{-a}|v|^{-a}|u-v|^{\gamma}|\xi|^{b}|\eta|^{b}|2 v-\xi-\eta|^{\frac{n-2}{2}}|v-\xi|^{\frac{1}{2}}} d \eta d \xi d v d u
\end{aligned}
$$

where we have used the change of variables (2.9) and the substitution (2.11), and $\gamma=(n-1)(1 / 2-1 / q)$ as before. We notice that the condition $\eta<\xi<v<u$ holds in the domain of integration above. From this condition we have the estimate

$$
\frac{|\xi-\eta|^{\frac{n-1}{q}}}{|2 v-\xi-\eta|^{\frac{n-2}{2}}|v-\xi|^{\frac{1}{2}}} \leq \frac{1}{|v-\xi|^{\gamma}} .
$$

In fact, (3.3) is equivalent to

$$
(\xi-\eta)^{\frac{n-1}{q}}(v-\xi)^{\gamma-\frac{1}{2}} \leq(2 v-\xi-\eta)^{\frac{n-2}{2}},
$$

and this follows from $\gamma>1 / 2, \xi<2 v-\xi, v-\eta>0$, and

$$
\frac{n-1}{q}+\left(\gamma-\frac{1}{2}\right)=\frac{n-2}{2}
$$


By (3.3) we have

$$
I_{2} \leq C \int_{0}^{\infty} \int_{0}^{v} \frac{1}{|v|^{-a}|v-\xi|^{\gamma}|\xi|^{b}}\left(\int_{2 v}^{\infty} \int_{-\xi}^{\xi} \frac{G(\xi, \eta) H(u, v)}{|u|^{-a}|u-v|^{\gamma}|\eta|^{b}} d \eta d u\right) d \xi d v
$$

If we prove

$$
\int_{2 v}^{\infty} \int_{-\xi}^{\xi} \frac{G(\xi, \eta) H(u, v)}{|u|^{-a}|u-v|^{\gamma}|\eta|^{b}} d \eta d u \leq C\|G(\xi, \cdot)\|_{L^{q^{\prime}}}\|H(\cdot, v)\|_{L^{q^{\prime}}}
$$

for $0<\xi<v$, then the estimate of $I_{2}$ finishes by applying Lemma 2.2.

In what follows we prove (3.4). By $0<\xi<v$ and the Hölder inequality, the left hand side of (3.4) is bounded by

$$
\begin{aligned}
& \left(\int_{2 v}^{\infty} \frac{H(u, v)}{|u|^{-a}|u-v|^{\gamma}} d u\right)\left(\int_{-v}^{v} \frac{G(\xi, \eta)}{|\eta|^{b}} d \eta\right) \\
& \leq\left(\int_{2 v}^{\infty} \frac{d u}{|u|^{-a q}|u-v|^{q \gamma}}\right)^{\frac{1}{q}}\|H(\cdot, v)\|_{L^{q^{\prime}}}\left(\int_{-v}^{v} \frac{d \eta}{|\eta|^{b q}}\right)^{\frac{1}{q}}\|G(\xi, \cdot)\|_{L^{q^{\prime}}} .
\end{aligned}
$$

We notice that

$$
-a q+q \gamma=q(2 / q-b)>1, \quad b q<1,
$$

since $a-b+\frac{n+1}{2}=\frac{n-1}{2}, b<1 / q$. Thus, integrals in (3.5) converge and (3.5) equals to a constant multiple of

$$
v^{a-\gamma+\frac{1}{q}}\|H(\cdot, v)\|_{L^{q^{\prime}}} v^{-b+\frac{1}{q}}\|G(\xi, \cdot)\|_{L^{q^{\prime}}},
$$

which implies (3.4) since $(a-\gamma+1 / q)+(-b+1 / q)=0$ by our assumption.

Estimate of $I_{3}$. In this case we apply the estimate on $\widetilde{K}_{\frac{n-2}{2}}$ in Lemma 3.1 with $\sigma=\gamma / 2$, where $\gamma=(n-1)(1 / 2-1 / q)$ as before. Note that $0<\sigma<1 / 2$, since $\frac{2(n-1)}{n-2}<q<\frac{2(n+1)}{n-1}$. Then,

$$
\begin{aligned}
I_{3} \leq C \int_{0}^{\infty} & \int_{0}^{t / 3} \int_{0}^{t-r} \int_{0}^{t-s-r} r^{\frac{n-1}{2}+\frac{\gamma}{2}} \lambda^{n-1}\left|t^{2}-r^{2}\right|^{a} \\
& \times(t-s-r)^{-\frac{n-2}{2}-\frac{\gamma}{2}}(t-s-r-\lambda)^{-\frac{1}{2}}|F(s, \lambda)||\Phi(t, r)| d \lambda d s d r d t \\
=C \int_{0}^{\infty} & \int_{u / 2}^{u} \int_{0}^{v} \int_{-\xi}^{\xi} \\
& \times \frac{|\xi-\eta|^{\frac{n-1}{q}} G(\xi, \eta) H(u, v)}{|u|^{-a}|v|^{-a}|u-v|^{\frac{\gamma}{2}}|\xi|^{b}|\eta|^{b}|2 v-\xi-\eta|^{\frac{n-2}{2}+\frac{\gamma}{2}}|v-\xi|^{\frac{1}{2}}} d \eta d \xi d v d u
\end{aligned}
$$

where we have used the change of variables (2.9) and the substitution (2.11). We notice that the condition $\eta<\xi<v<u$ holds in the domain of integration above. 
Form this condition we have the estimate

$$
\frac{1}{|2 v-\xi-\eta|^{\frac{n-2}{2}+\frac{\gamma}{2}}} \leq \frac{1}{|\xi-\eta|^{\frac{n-1}{q}}|v-\eta|^{\frac{\gamma}{2}}|v-\xi|^{\gamma-\frac{1}{2}}},
$$

since $2 v-\xi>\xi, v-\xi>0, v-\eta>0$, and

$$
\frac{n-2}{2}+\frac{\gamma}{2}=\frac{n-1}{q}+\frac{\gamma}{2}+\left(\gamma-\frac{1}{2}\right)
$$

Note that $2 v-\xi-\eta>0$. By (3.6) and changing the order of integrals with respect to integral variables,

$$
\begin{aligned}
I_{3} \leq C \int_{0}^{\infty} \int_{0}^{v} & \frac{1}{|v|^{-a}|v-\xi|^{\gamma}|\xi|^{b}} \\
& \times\left(\int_{v}^{2 v} \int_{-\xi}^{\xi} \frac{G(\xi, \eta) H(u, v)}{|u|^{-a}|u-v|^{\frac{\gamma}{2}}|v-\eta|^{\frac{\gamma}{2}}|\eta|^{b}} d \eta d u\right) d \xi d v
\end{aligned}
$$

If we prove

$$
\int_{v}^{2 v} \int_{-\xi}^{\xi} \frac{G(\xi, \eta) H(u, v)}{|u|^{-a}|u-v|^{\frac{\gamma}{2}}|v-\eta|^{\frac{\gamma}{2}}|\eta|^{b}} d \eta d u \leq C\|G(\xi, \cdot)\|_{L^{q^{\prime}}}\|H(\cdot, v)\|_{L^{q^{\prime}}}
$$

for $0<\xi<v$, then the estimate of $I_{3}$ finishes by applying Lemma 2.2.

In what follows we prove (3.7). By $0<\xi<v$ and the Hölder inequality, the left hand side of (3.7) is bounded by

$$
\begin{aligned}
& \left(\int_{v}^{2 v} \frac{H(u, v)}{|u|^{-a}|u-v|^{\frac{\gamma}{2}}} d u\right)\left(\int_{-v}^{v} \frac{G(\xi, \eta)}{|v-\eta|^{\frac{\gamma}{2}}|\eta|^{b}} d \eta\right) \\
& \leq\left(\int_{v}^{2 v} \frac{d u}{|u|^{-a q}|u-v|^{\frac{q \gamma}{2}}}\right)^{\frac{1}{q}}\|H(\cdot, v)\|_{L^{q^{\prime}}}\left(\int_{-v}^{v} \frac{d \eta}{|v-\eta|^{\frac{q \gamma}{2}}|\eta|^{b q}}\right)^{\frac{1}{q}}\|G(\xi, \cdot)\|_{L^{q^{\prime}}} .
\end{aligned}
$$

The above integrals converge since

$$
-a q<1, \quad b q<1, \quad q r / 2<1,
$$

which are due to $b>\frac{n}{q}-\frac{n-1}{2}$ with $a-b+\frac{n+1}{q}=\frac{n+1}{2}, b<\frac{1}{q}, q<\frac{2(n+1)}{n-1}$, respectively. We notice that by the third condition in (3.9) the end point $q=\frac{2(n+1)}{n-1}$ is excluded. Thus, the right hand side of (3.8) is a constant multiple of

$$
v^{a-\frac{\gamma}{2}+\frac{1}{q}}\|H(\cdot, v)\|_{L^{q^{\prime}}} v^{-\frac{\gamma}{2}-b+\frac{1}{q}}\|G(\xi, \cdot)\|_{L^{q^{\prime}}},
$$

which implies (3.7) since $a-\frac{\gamma}{2}+\frac{1}{q}+\left(-\frac{\gamma}{2}-b+\frac{1}{q}\right)=0$.

This completes the proof of Theorem 2 in the case where $n \geq 4, \frac{2(n-1)}{n-2}<q<$ $\frac{2(n+1)}{n-1}$. 


\section{Proof of Theorem 1 in the case $n=2$}

In this section we give the proof of Theorem 1 in the case $n=2$. In contrast with the case $n \geq 4$, we observe that $0<\gamma=(n-1)(1 / 2-1 / q)<1 / 3$ for $2<q<\frac{2(n-1)}{n+1}$ when $n=2$, and this is the reason we treat the case $n=2$ separately.

Proof of Theorem 2 in the case $n=2$. As in the preceding cases, it is sufficient to prove the estimate (2.7). Decomposing the right hand side of (2.8) further, we observe that

$$
\begin{aligned}
& \int_{0}^{\infty} \int_{0}^{\infty}\left|t^{2}-r^{2}\right|^{a} w(t, r) \Phi(t, r) r d r d t \\
& =\left(\int_{0}^{\infty} \int_{0}^{\infty} \int_{\max (t-r, 0)}^{t} \int_{r-(t-s)}^{r+t-s}\right. \\
& \left.\quad+\int_{0}^{\infty} \int_{0}^{t} \int_{0}^{t-r} \int_{\max \left(t-s-r, \frac{t-2 s+r}{2}\right)}^{t-s+r}+\int_{0}^{\infty} \int_{t / 3}^{t} \int_{0}^{t-r} \int_{t-s-r}^{\frac{t-2 s+r}{2}}\right) \\
& \quad+\int_{0}^{\infty} \int_{0}^{t} \int_{0}^{t-r} \int_{0}^{t-s-r} r \lambda\left|t^{2}-r^{2}\right|^{a} L(\lambda, r, t-s) F(s, \lambda) \Phi(t, r) d \lambda d s d r d t \\
& \equiv I_{1}+I_{2}+\left.I^{2}\right|^{a} \widetilde{L}(\lambda, r, t-s) F(s, \lambda) \Phi(t, r) d \lambda d s d r d t
\end{aligned}
$$

Note that $(t-2 s+r) / 2>t-s-r$ implies $r>t / 3$.

In what follows we prove

$$
\left|I_{j}\right| \leq C\left\|\left|t^{2}-r^{2}\right|^{b} r^{\frac{1}{q^{\prime}}} F\right\|_{L^{q^{\prime}}}\left\|r^{\frac{1}{q^{\prime}}} \Phi\right\|_{L^{q^{\prime}}}, \quad j=1,2,3,4
$$

to derive $(2.7)$ for $2<q<6$.

Estimate of $I_{1}$. The estimate of $I_{1}$ is derived similarly as in the estimate of $I_{1}$ in the case where $n \geq 4,2<q<\frac{2(n-1)}{n-2}$ in Section 2, and thus we omit the proof of the estimate of $I_{1}$ here.

Estimate of $I_{2}$. In this case, we apply the second estimate on $L$ of Lemma 2.1 with $\sigma=\gamma+\frac{4}{3}\left(\frac{1}{3}-\gamma\right)$, where $\gamma=1 / 2-1 / q$. We notice that $1 / 3<\sigma<4 / 9$ when $2<q<6$. Then,

$$
\begin{aligned}
\left|I_{2}\right| \leq C & \int_{0}^{\infty} \int_{0}^{t} \int_{0}^{t-r} \int_{\max \left(t-s-r, \frac{t-2 s+r}{2}\right)}^{t-s+r} r \lambda^{\frac{1}{2}+\sigma}\left|t^{2}-r^{2}\right|^{a} \\
& \times(r+\lambda-t+s)^{-\sigma}(r+\lambda+t-s)^{-\sigma}(t-s+r-\lambda)^{-\frac{1}{2}+\sigma} \\
& \times|F(s, \lambda)||\Phi(t, r)| d \lambda d s d r d t .
\end{aligned}
$$


Changing of variables (2.9) and using the substitution (2.11), we observe that the right hand side of (4.2) equals to a constant multiple of

$$
\begin{aligned}
& \left(\int_{0}^{\infty} \int_{u / 2}^{u} \int_{v}^{u} \int_{-\xi}^{-\xi+2 v}+\int_{0}^{\infty} \int_{0}^{u / 2} \int_{u / 2}^{u} \int_{-\xi}^{-\xi+2 v}\right) \\
& \quad \times \frac{|u-v|^{\frac{1}{2}-\gamma}|\xi-\eta|^{\sigma-\gamma} G(\xi, \eta) H(u, v)}{|u|^{-a}|v|^{-a}|\xi|^{b}|\eta|^{b}|\xi-v|^{\sigma}|u-\eta|^{\sigma}|u-\xi|^{\frac{1}{2}-\sigma}} d \eta d \xi d v d u
\end{aligned}
$$

In both domains of the integration above, the condition $-\xi<\eta<v<\xi<u, \xi>0$ holds. In fact, in the domain of the integration of the first term we have

$$
-\xi<\eta<-\xi+2 v<v<\xi<u
$$

and in the domain of the integration of the second term we have

$$
-\xi<\eta<-\xi+2 v<v<u / 2<\xi<u \text {. }
$$

To estimate the integral kernel we use the following claim.

Claim 3. If $-\xi<\eta<v<\xi<u, \xi>0$, then for $0<\gamma<1 / 3$

$$
\frac{|u-v|^{\frac{1}{2}-\gamma}|\xi-\eta|^{\sigma-\gamma}}{|\xi-v|^{\sigma}|u-\eta|^{\sigma}|u-\xi|^{\frac{1}{2}-\sigma}} \leq \frac{|\xi|^{\sigma-\gamma}|u|^{1-\gamma-2 \sigma}}{|\xi-v|^{\sigma}|u-\xi|^{\frac{1}{2}-\sigma}|\xi-\eta|^{\frac{1}{2}-\sigma}},
$$

where $\sigma=\gamma+\frac{4}{3}\left(\frac{1}{3}-\gamma\right)$.

Proof. We first observe that

$$
\frac{(u-v)^{\frac{1}{2}-\gamma}}{(u-\eta)^{\sigma}}=\frac{(u-v)^{\frac{1}{2}-\gamma}}{(u-\eta)^{\frac{1}{2}-\sigma}(u-\eta)^{2 \sigma-\frac{1}{2}}} \leq \frac{(u-v)^{1-\gamma-2 \sigma}}{(u-\eta)^{\frac{1}{2}-\sigma}},
$$

since $u-\eta>u-v$ and $2 \sigma-1 / 2>0$. Then we have

$$
\frac{|u-v|^{\frac{1}{2}-\gamma}|\xi-\eta|^{\sigma-\gamma}}{|\xi-v|^{\sigma}|u-\eta|^{\sigma}|u-\xi|^{\frac{1}{2}-\sigma}} \leq \frac{(\xi-\eta)^{\sigma-\gamma}(u-v)^{1-\gamma-2 \sigma}}{(\xi-v)^{\sigma}(u-\eta)^{\frac{1}{2}-\sigma}(u-\xi)^{\frac{1}{2}-\sigma}} .
$$

Here, we notice that $1-\gamma-2 \sigma=\frac{1}{3}\left(\frac{1}{3}-\gamma\right)>0, \sigma-\gamma=\frac{4}{3}\left(\frac{1}{3}-\gamma\right)>0$. Thus we obtain

$$
(u-v)^{1-\gamma-2 \sigma} \leq u^{1-\gamma-2 \sigma}, \quad(\xi-\eta)^{\sigma-\gamma} \leq(2 \xi)^{\sigma-\gamma},
$$

since $u>v>0,-\eta<\xi, \xi>0$ and this completes the proof of Claim 3. 
Thus, applying Claim 3 and changing the order of integrals with respect to integral variables, we obtain

$$
\begin{aligned}
\left|I_{2}\right| \leq C\left(\int_{0}^{\infty}\right. & \left.\int_{v}^{2 v} \int_{\xi}^{2 v} \int_{-\xi}^{-\xi+2 v}+\int_{0}^{\infty} \int_{v}^{2 v} \int_{v}^{2 \xi} \int_{-\xi}^{-\xi+2 v}+\int_{0}^{\infty} \int_{2 v}^{\infty} \int_{\xi}^{2 \xi} \int_{-\xi}^{-\xi+2 v}\right) \\
& \times \frac{|u|^{1-\gamma-2 \sigma}|\xi|^{\sigma-\gamma} G(\xi, \eta) H(u, v)}{|u|^{-a}|v|^{-a}|\xi|^{b}|\eta|^{b}|\xi-v|^{\sigma}|u-\xi|^{\frac{1}{2}-\sigma}|\xi-\eta|^{\frac{1}{2}-\sigma}} d \eta d u d \xi d v \\
\leq C \int_{0}^{\infty} & \int_{v}^{\infty} \frac{1}{|v|^{-a}|v-\xi|^{\sigma}|\xi|^{b-\sigma+\gamma}} \\
& \times\left(\int_{\xi / 2}^{2 \xi} \int_{-\xi}^{\xi} \frac{G(\xi, \eta) H(u, v)}{|u|^{-a+\gamma+2 \sigma-1}|u-\xi|^{\frac{1}{2}-\sigma}|\xi-\eta|^{\frac{1}{2}-\sigma}|\eta|^{b}} d \eta d u\right) d \xi d v .
\end{aligned}
$$

For $\xi>0$, we observe that

$$
\begin{aligned}
& \int_{\xi / 2}^{2 \xi} \int_{-\xi}^{\xi} \frac{G(\xi, \eta) H(u, v)}{|u|^{-a+\gamma+2 \sigma-1}|u-\xi|^{\frac{1}{2}-\sigma}|\xi-\eta|^{\frac{1}{2}-\sigma}|\eta|^{b}} d \eta d u \\
& =\left(\int_{\xi / 2}^{2 \xi} \frac{H(u, v)}{|u|^{-a+\gamma+2 \sigma-1}|u-\xi|^{\frac{1}{2}-\sigma}} d u\right)\left(\int_{-\xi}^{\xi} \frac{G(\xi, \eta)}{|\xi-\eta|^{\frac{1}{2}-\sigma}|\eta|^{b}} d \eta\right) \\
& \leq\left(\int_{\xi / 2}^{2 \xi} \frac{d u}{|u|^{q(-a+\gamma+2 \sigma-1)}|u-\xi|^{q\left(\frac{1}{2}-\sigma\right)}} d u\right)^{\frac{1}{q}}\|H(\cdot, v)\|_{L^{q^{\prime}}} \\
& \times\left(\int_{-\xi}^{\xi} \frac{d \eta}{|\xi-\eta|^{q\left(\frac{1}{2}-\sigma\right)}|\eta|^{b q}} d \eta\right)^{\frac{1}{q}}\|G(\xi, \cdot)\|_{L^{q^{\prime}}} \\
& =C\|H(\cdot, v)\|_{L^{q^{\prime}}}\|G(\xi, \cdot)\|_{L^{q^{\prime}}} .
\end{aligned}
$$

Here, we notice that

$$
q\left(\frac{1}{2}-\sigma\right)=\frac{1}{3}\left(\frac{2}{3} q-1\right)<1, \quad b q<1
$$

since $q<6, b<1 / q$, and

$$
\left(a-\gamma-2 \sigma+1-\frac{1}{2}+\sigma+\frac{1}{q}\right)+\left(-\frac{1}{2}+\sigma-b+\frac{1}{q}\right)=0 .
$$

Thus, we obtain

$$
\left|I_{2}\right| \leq C \int_{\mathbf{R}} \int_{\mathbf{R}} \frac{\|H(\cdot, v)\|_{L^{q^{\prime}}}\|G(\xi, \cdot)\|_{L^{q^{\prime}}}}{|v|^{-a}|v-\xi|^{\sigma}|\xi|^{b-\sigma+\gamma}} d \xi d v
$$

Finally, applying Lemma 2.2 we obtain (4.1) for $j=2$, since

$$
b-\sigma+\gamma=b-\frac{4}{3}\left(\gamma-\frac{1}{3}\right)<b<\frac{1}{q}, \quad-a+(b-\sigma+\gamma)=\frac{5}{3}\left(\frac{1}{q}-\frac{1}{6}\right)>0,
$$


and other assumptions easily follow.

Estimate of $I_{3}$. Applying the second estimate on $L$ of Lemma 2.1 with $\sigma=\gamma$, we obtain

$$
\begin{aligned}
&\left|I_{3}\right| \leq C \int_{0}^{\infty} \int_{t / 3}^{t} \int_{0}^{t-r} \int_{t-s-r}^{(t-2 s+r) / 2} r \lambda^{\frac{1}{2}+\gamma}\left|t^{2}-r^{2}\right|^{a} \\
& \times(r+\lambda-t+s)^{-\gamma}(r+\lambda+t-s)^{-\gamma}(t-s+r-\lambda)^{-\frac{1}{2}+\gamma} \\
& \times|F(s, \lambda)||\Phi(t, r)| d \lambda d s d r d t \\
& \leq C \int_{0}^{\infty} \int_{t / 3}^{t} \int_{0}^{t-r} \int_{t-s-r}^{(t-2 s+r) / 2} r^{\frac{1}{2}+\gamma} \lambda^{\frac{1}{2}+\gamma}\left|t^{2}-r^{2}\right|^{a} \\
& \quad \times(r+\lambda-t+s)^{-\gamma}(r+\lambda+t-s)^{-\gamma}|F(s, \lambda)||\Phi(t, r)| d \lambda d s d r d t
\end{aligned}
$$

since $0<\gamma<1 / 3$, and $(t-2 s+r) / 2>\lambda$ implies $t-s+r-\lambda>r / 2$, where $\gamma=1 / 2-1 / q$. Here, we notice that $2<q<6$ in this case. Changing of variables (2.9) and using the substitution (2.11), we observe that

$$
\begin{aligned}
\left|I_{3}\right| & \leq C \int_{0}^{\infty} \int_{0}^{u / 2} \int_{v}^{u / 2} \int_{-\xi}^{-\xi+2 v} \frac{G(\xi, \eta) H(u, v)}{|u|^{-a}|v|^{-a}|\xi|^{b}|\eta|^{b}|\xi-v|^{\gamma}|u-\eta|^{\gamma}} d \eta d \xi d v d u \\
& \leq C \int_{\mathbf{R}} \int_{\mathbf{R}} \frac{1}{|v|^{-a}|v-\xi|^{\gamma}|\xi|^{b}}\left(\int_{\mathbf{R}} \int_{\mathbf{R}} \frac{G(\xi, \eta) H(u, v)}{|u|^{-a}|u-\eta|^{\gamma}|\eta|^{b}} d \eta d u\right) d \xi d v .
\end{aligned}
$$

Thus, applying Lemma 2.2 we obtain (4.1) for $j=3$.

Estimate of $I_{4}$. Applying the estimate on $\widetilde{L}$ of Lemma 2.1 with $\sigma=\gamma$, we obtain

$$
\begin{aligned}
\left|I_{4}\right| \leq C \int_{0}^{\infty} & \int_{0}^{t} \int_{0}^{t-r} \int_{0}^{t-s-r} r^{\frac{1}{2}+\gamma} \lambda^{\frac{1}{2}+\gamma}\left|t^{2}-r^{2}\right|^{a} \\
& \times(t-s-r-\lambda)^{-\gamma}(t-s+r+\lambda)^{-\gamma}|F(s, \lambda)||\Phi(t, r)| d \lambda d s d r d t .
\end{aligned}
$$

Changing of variables (2.9) and using the substitution (2.11), we observe that

$$
\left|I_{4}\right| \leq C \int_{0}^{\infty} \int_{0}^{u} \int_{0}^{v} \int_{-\xi}^{\xi} \frac{G(\xi, \eta) H(u, v)}{|u|^{-a}|v|^{-a}|\xi|^{b}|\eta|^{b}|\xi-v|^{\gamma}|u-\eta|^{\gamma}} d \eta d \xi d v d u
$$

and then $\left|I_{4}\right|$ is also bounded by the right hand side of (4.3). Thus, applying Lemma 2.2 we obtain (4.1) for $j=4$.

This completes the proof of Theorem 2 in the case $n=2$.

\section{References}

[1] V. Georgiev, H. Lindblad, C. D. Sogge, Weighted Strichartz estimates and global existence for semilinear wave equations, Amer. J. Math. 119 (1997), 1291-1319. 
[2] K. Hidano, Scattering and self-similar solutions for the nonlinear wave equations, Differential Integral Equations 15 (2002), 405-462.

[3] J. Kato, T. Ozawa, On solutions of the wave equation with homogeneous Cauchy data, to appear in Asymptotic Anal.

[4] J. Kato, T. Ozawa, Weighted Strichartz estimates and existence of self-similar solutions for semilinear wave equations, to appear in Indiana Univ. Math. J. (Announcement appeared in "Tosio Kato's Method and Principle for Evolution Equations in Mathematical Physics," (H. Fujita, S. T. Kuroda, H. Okamoto Eds.), Yurinsha (2002), 227-238.)

[5] H. Kubo, K. Kubota, Asymptotic behaviors of radially symmetric solutions of $\square u=|u|^{p}$ for super critical values $p$ in even space dimensions, Japan. J. Math. 24 (1998), 191-256.

[6] K. Mochizuki, T. Motai, The scattering theory for the nonlinear wave equation with small data, J. Math. Kyoto Univ. 25 (1985), 703-715.

[7] H. Pecher, Self-similar and asymptotically self-similar solutions of nonlinear wave equations, Math. Ann. 316 (2000), 259-281.

[8] H. Pecher, Sharp existence results for self-similar solutions of semilinear wave equations, NoDEA Nonlinear Differential Equations Appl. 7 (2000), 323-341.

[9] F. Planchon, Self-similar solutions and semi-linear wave equations in Besov spaces, J. Math. Pures Appl. 79 (2000), 809-820.

[10] M. A. Rammaha, Finite-time blow-up for nonlinear wave equations in high dimensions, Comm. Partial Differential Equations, 12 (1987), 677-700.

[11] F. Ribaud, A. Youssfi, Solutions globales et solutions auto-similaires de l'équation des ondes non linéaire, C. R. Acad. Sci. Paris, Série I Math. 329 (1999), 33-36.

[12] E. M. Stein, G. Weiss, Fractional integrals on $n$-dimensional Euclidean space, J. Math. Mech. 7 (1958), 503-514.

[13] T. Takamura, Blow-up for semilinear wave equations with slowly decaying data in high dimensions, Differential Integral Equations 8 (1995), 647-661. 
[14] D. Tataru, Strichartz estimates in the hyperbolic space and global existence for the semilinear wave equation, Trans. Amer. Math. Soc. 353 (2001), 795-807. 